\title{
First-order logics: some characterizations and closure properties
}

\author{
Christian Choffrut · Andreas Malcher \\ Carlo Mereghetti · Beatrice Palano
}

Received: date / Accepted: date

\begin{abstract}
The characterization of the class of $\mathrm{FO}[+]$-definable languages by some generating or recognizing device is still an open problem. We prove that, restricted to word bounded languages, this class coincides with the class of semilinear languages. We also study the closure properties of the classes of languages definable in $\mathrm{FO}[+1], \mathrm{FO}[<], \mathrm{FO}[+]$ and $\mathrm{FOC}[+]$ under the main classical operations.
\end{abstract}

Keywords Word bounded languages · Semilinear sets · First-order logic · Counting quantifier $\cdot$ Shuffle $\cdot$ Concatenation

\section{Introduction}

The aim of descriptive complexity is to provide logical characterizations of relevant classes of languages. The first result in this area dates back to Büchi [4] who gave a characterization of regular languages via monadic second order logic. Since then, a well consolidated trend in the literature provides characterizations of several language classes via different logics. This has also other consequences: a logical description of a language often leads to a precise estimate of the parallel complexity of membership and related problems for that language (see, e.g., $[1,18])$.

This work was partially supported by the Italian MURST under the project "PRIN: Aspetti matematici e applicazioni emergenti degli automi e dei linguaggi formali: metodi probabilistici e combinatori in ambito di linguaggi formali", and by CRUI/DAAD under the project "Programma Vigoni: Reducing complexity by introducing structure".

A preliminary version has been published in the Proceedings of the 4th International Conference on Language and Automata Theory and Applications (LATA 2010).

Christian Choffrut

LIAFA, UMR 7089, 175 Rue du Chevaleret, Paris 13, France

E-mail: fcc@liafa.jussieu.fr

Andreas Malcher

Institut für Informatik, Universität Giessen, Arndtstr. 2, 35392 Giessen, Germany

E-mail: malcher@informatik.uni-giessen.de

Carlo Mereghetti · Beatrice Palano

DSI, Università degli Studi di Milano, via Comelico 39/41, 20135 Milano, Italy

E-mail: \{mereghetti, palano\}@dsi.unimi.it 
Important first-order logics for language description are $\mathrm{FO}[+1], \mathrm{FO}[<]$ and $\mathrm{FO}[+]$. All these logics are used to express properties of words, and their variables range over word positions. Along with the usual predicates $Q_{a}(x)$ holding true whenever the letter at position $x$ is $a$, and equality, they are provided with the predicates $x+1=y, x<y$ and $x+y=z$, respectively. A further step in incrementing the descriptive power was made two decades ago by introducing the notion of counting quantifier (see, e.g., $[1,6,17]$ ) which roughly speaking enables us to count the number of values satisfying a given formula. These new quantifiers were modeled after the majority function used in circuit complexity and may not be confused with the modular quantifiers whose descriptive power is much weaker.

It is well-known (see, e.g., [18]) that $\mathrm{FO}[+1]$ characterizes the class of locally threshold testable languages, while $\mathrm{FO}[<]$ characterizes the wider class of star-free languages, this latter class being itself strictly contained in that of regular languages. The class of languages described in $\mathrm{FO}[+]$ contains all the star-free languages, not all the regular languages and also contains nonregular languages. Presently, no precise definition of the class of languages characterized by $\mathrm{FO}[+]$ is known. Thus, it is natural to investigate the possibility of representing relevant subclasses. Our first result concerns the important subclass of the bounded languages which are definable in $\mathrm{FO}[+]$, based on the well-known notion of semilinear languages introduced by Ginsburg and Spanier in 1964 [9].

Theorem. A bounded language is definable in $\mathrm{FO}[+]$ if and only if it is semilinear.

This is particularly interesting since such a logical characterization of bounded semilinear languages complements the known characterizations by formal grammars (e.g., simple matrix grammars [11]) and automata (e.g., certain variants of multi-head finite automata and multi-head pushdown automata [12]).

The second part of this paper is concerned with another type of issue which, we think, was only marginally considered by people working in parallel complexity and which is more relevant in the theory of languages. Indeed, we investigate the closure properties of the classes of languages defined in various logics, see Figure 1, where FOC $[+]$ refers to $\mathrm{FO}[+]$ augmented with counting quantifiers. Some entries of the table were already known, at least implicitly. Two remain unanswered.

Table 1 Closure properties.

\begin{tabular}{lllll}
\hline & FO $[+1]$ & FO $[<]$ & FO $[+]$ & FOC $[+]$ \\
\hline \hline length-preserving morphism & no & no & no & no \\
\hline length-preserving inverse morphism & yes & yes & yes & yes \\
\hline inverse morphism & no & yes & no & yes \\
\hline concatenation & no & yes & yes & yes \\
\hline shuffle & no & no & no & $?$ \\
\hline disjoint shuffle & no & yes & no & yes \\
\hline Kleene star & no & no & no & yes if $\mathrm{TC}^{0}=\mathrm{NC}^{1}$ \\
\hline quotient & yes & yes & yes & yes \\
\hline conjugate image & yes & yes & yes & yes \\
\hline commutative image & no & no & no & $?$ \\
\hline reversal & yes & yes & yes & yes \\
\hline
\end{tabular}

Our characterization and closure properties lead us to results on the logical definability of the meaningful class of the Dyck languages [5,10]. It is known from [1] that the Dyck 
languages can be described by FOC $[+]$. Moreover, from [14], one may easily get that the Dyck languages cannot be described in $\mathrm{FO}[+]$. Here, we give a new proof of this latter result relying on logics.

\section{Preliminaries}

The set of natural numbers is here denoted by $\mathbb{N}$. We assume basic notions on formal language theory [10]. Given an alphabet $\Sigma$, we denote by $\Sigma^{*}$ the set of words on $\Sigma$, including the empty word $\varepsilon$. We denote by $|x|$ the length of a word $x \in \Sigma^{*}$ and by $\Sigma^{i}$ the set of words of length $i$, with $\Sigma^{0}=\{\varepsilon\}$. We let $\Sigma^{+}=\Sigma^{*} \backslash\{\varepsilon\}$. For any $x, w \in \Sigma^{*}$, we let $|x|_{w}$ be the number of occurrences of the word $w$ in $x$. A language on $\Sigma$ is any subset of $\Sigma^{*}$.

We assume familiarity with traditional and threshold circuits as computational models to study the parallel complexity of problems (see, e.g., $[15,19])$. We recall that $\mathrm{NC}^{k}\left(\mathrm{AC}^{k}\right)$ is the class of problems solved by families of bounded (unbounded) fan-in AND/OR/NOTcircuits of polynomial size and $O\left(\log ^{k} n\right)$ depth. The class $\mathrm{TC}^{0}$ contains those problems solved by constant depth threshold circuits of polynomial size. We have the inclusions $\mathrm{AC}^{0} \subset \mathrm{TC}^{0} \subseteq \mathrm{NC}^{1}$, and the latter inclusion is widely considered to be proper. It is known that regular languages lie in $\mathrm{NC}^{1}$ [16], but not in $\mathrm{TC}^{0}$ unless $\mathrm{TC}^{0}=\mathrm{NC}^{1}$ [3]. In [13], further interesting subclasses of context-free languages are shown to be in $\mathrm{NC}^{1}$, such as the class of the Dyck languages over an arbitrary number of parentheses, and the class of bounded semilinear languages (see Sections 3 and 5 for a definition of these languages).

The connection between circuit complexity issues and first-order logic formalisms for language description is extensively covered in [18]. In these formalisms, the words over $\Sigma$ are represented as first-order structures in the signature $\left\langle\left\{Q_{a}\right\}_{a \in \Sigma},\left\{P_{i}\right\}_{1<i<m}\right.$, last $\rangle$, so that the structure for a word $w$ of length $n$ has universe $\{1, \ldots, n\}, Q_{a}$ is the unary predicate holding true for $1 \leq j \leq n$ if and only if the $j$ th letter of $w$ is $a$, the $P_{i}$ 's are numerical predicates of different arities (e.g., $x<y$ or $x=y+z$ ) and last is the constant $n$. In fact, all logics considered in the sequel assume the predicates $Q_{a}$, the numerical predicate $x=y$, and the constant last. Yet, they differ on the set $\mathscr{Z}$ of the assumed additional numerical predicates, e.g.: +1 for the immediate successor, $<$ for the usual ordering on the nonnegative integers, and + for the ternary predicate $x=y+z$. In this way, we use the notation $\mathrm{FO}[\mathscr{Z}]$, where FO stands for first-order quantification. The formulas from $\mathrm{FO}[\mathscr{Z}]$ are defined in the usual way, i.e.: every atomic predicate is a formula; if $\varphi_{1}$ and $\varphi_{2}$ are formulas, then $\varphi_{1} \wedge \varphi_{2}, \varphi_{1} \vee \varphi_{2}$ and $\neg \varphi_{1}$ are formulas; if $\varphi\left(x_{1}, \ldots, x_{n}\right)$ is a formula whose free variables are $x_{1}, \ldots, x_{n}$, then $\exists x_{i} \varphi\left(x_{1}, \ldots, x_{n}\right)$ and $\forall x_{i} \varphi\left(x_{1}, \ldots, x_{n}\right)$, with $1 \leq i \leq n$, are formulas.

The notion of counting quantifiers is less known and deserves a precise definition. We restrict ourselves to the unary version. Given a formula $\phi\left(x_{1}, \ldots, x_{n}\right)$ and a variable $y$, $\exists_{x_{i}}^{y} \phi\left(x_{1}, \ldots, x_{n}\right)$ is a formula with free variables $y, x_{1}, \ldots, x_{i-1}, x_{i+1}, \ldots, x_{n}$. It is true for the assignment $b$ to $y$ and $a_{j}$ to $x_{j}, j \neq i$, if there exist $b>0$ values $a$ for $x_{i}$ such that $\phi\left(a_{1}, \ldots, a_{i-1}, a, a_{i+1}, \ldots, a_{n}\right)$ holds true. In other words it is equivalent to the formula

$$
(1 \leq y \leq \text { last }) \wedge\left(y=\left|\left\{x \mid \phi\left(x_{1}, \ldots, x_{i-1}, x, x_{i+1}, \ldots, x_{n}\right)\right\}\right|\right) .
$$

In particular, it there exists no such $a$, then the formula is a contradiction.

Formulas are meant to specify languages. Indeed, if $\varphi$ is a sentence (a formula without free variables), we let $L_{\varphi}$ be the set of all words satisfying $\varphi$. Formally, we write $L_{\varphi}=$ $\left\{w \in \Sigma^{*} \mid w=\varphi\right\}$. In this case, we say that $L_{\varphi}$ is the language defined (or described) by $\varphi$. We denote by $\mathscr{L}(\mathrm{FO}[\mathscr{Z}])$ the class of languages definable in $\mathrm{FO}[\mathscr{Z}]$, i.e., $\mathscr{L}(\mathrm{FO}[\mathscr{Z}])=$ 
$\left\{L \subseteq \Sigma^{*} \mid L=L_{\varphi}\right.$, for some sentence $\left.\varphi \in \mathrm{FO}[\mathscr{Z}]\right\}$. Several classes of languages have been logically characterized. For instance, $\mathrm{FO}[+1]$ (resp., $\mathrm{FO}[<]$ ) is the first-order logic with numerical predicate +1 (resp., $<$ ). It is well-known that $\mathscr{L}(\mathrm{FO}[+1])$ is the class of locally threshold testable languages, while $\mathscr{L}(\mathrm{FO}[<])$ is the class of star-free languages. No formal language characterization for $\mathscr{L}(\mathrm{FO}[+])$ is currently known. We denote by FOC the set of first-order quantifiers together with the counting quantifier ' $\exists$ '. In the sequel, we use the predicate symbol ' $*$ ' for the natural multiplication. The following strict inclusions are well-known (see [17], for more details):

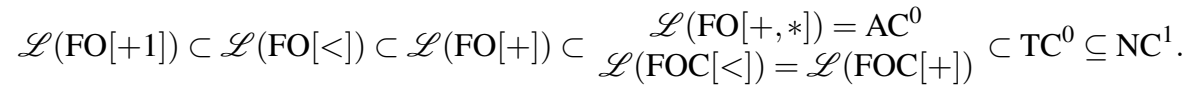

\section{Bounded Languages}

In this section, we exhibit a relevant class of languages contained in $\mathscr{L}(\mathrm{FO}[+])$, and show that, restricted to bounded languages, $\mathrm{FO}[+]$ characterizes the semilinear languages.

We recall that a set $X \subseteq \mathbb{N}^{m}$ is linear whenever, for some integer $r \in \mathbb{N}$, there exist vectors $v_{0}, \ldots, v_{r} \in \mathbb{N}^{m}$ such that $X=v_{0}+\sum_{t=1}^{r} \mathbb{N} v_{t}$. A semilinear set is a finite union of linear sets. Let $w_{1}, \ldots, w_{m}$ be a sequence of words in $\Sigma^{*}$ where the same word may have several occurrences. We consider the natural embedding of $\mathbb{N}^{m}$ into $\Sigma^{*}$ (we shall simply say "the natural embedding") relative to this sequence $w_{1}, \ldots, w_{m}$ as the mapping $\chi: \mathbb{N}^{m} \rightarrow \Sigma^{*}$ defined by $\chi\left(n_{1}, \ldots, n_{m}\right)=w_{1}^{n_{1}} \cdots w_{m}^{n_{m}}$. A language $L \subseteq \Sigma^{*}$ is bounded whenever $L=\chi(X)$ holds for some $X \subseteq \mathbb{N}^{m}$. It is letter bounded whenever all $w_{i}$ 's are letters. Moreover, $L$ is bounded (linear) semilinear whenever $X$ is a (linear) semilinear set. Rigorously speaking, we should always specify the sequence $w_{1}, \ldots, w_{m}$ with the mapping $\chi$. However, the context should clearly determine which sequence is assumed.

We first prove the result under the hypothesis that the language is letter bounded, and then we extend it to arbitrary bounded languages.

\subsection{A preliminary reduction}

Notice that, without loss of generality, we may assume $w_{i} \neq w_{i+1}$ for every $1 \leq i<m$. Indeed, it clearly suffices to consider the case where $X$ is linear, i.e., of the form $v_{0}+\sum_{t=1}^{r} \mathbb{N} v_{t}$. Suppose there exists $1 \leq i<m$ such that $w_{i}=w_{i+1}$, and denote with $v_{t, j}$ the $j$ th component of the vector $v_{t} \in \mathbb{N}^{m}$. With each $v_{t}$, for $1 \leq t \leq r$, we associate the vector $v_{t}^{\prime} \in \mathbb{N}^{m-1}$ defined by

$$
v_{t, j}^{\prime}= \begin{cases}v_{t, j} & \text { if } j<i \\ v_{t, j}+v_{t, j+1} & \text { if } j=i \\ v_{t, j+1} & \text { otherwise }\end{cases}
$$

By letting $\chi^{\prime}$ be the natural embedding of $\mathbb{N}^{m-1}$ into $w_{1}^{*} \cdots w_{i}^{*} w_{i+2}^{*} \cdots w_{m}^{*}$, we get $L=\chi^{\prime}\left(X^{\prime}\right)$ with the linear set $X^{\prime}=v_{0}^{\prime}+\sum_{t=1}^{r} \mathbb{N} v_{t}^{\prime}$.

Some closure properties of the bounded semilinear languages are obtained as technical adaptations of the closure properties of the semilinear sets in $\mathbb{N}^{m}$, as we shall discuss in Proposition 1. The following is a technical property which deals with the problem of the occurrence of the empty word among $w_{1}, \ldots, w_{m}$. 
Lemma 1 Every bounded semilinear subset of $\Sigma^{*}$ is a finite union of linear subsets in $w_{1}^{+} \cdots w_{m}^{+}$, with $w_{i} \neq \varepsilon$ for $i=1, \ldots, m$, and possibly of the subset containing only the empty word.

Proof Indeed, the sets of the form $M_{1} \times \cdots \times M_{m}$, where $M_{i}=\{0\}$ or $M_{i}=\mathbb{N} \backslash\{0\}$, are linear and thus so are all intersections $X \cap M_{1} \times \cdots \times M_{m}$, with $X$ a linear set. Let $\emptyset \subset I \subseteq\{1, \ldots, m\}$ be the set of indices $i$ such that $M_{i}=\mathbb{N} \backslash\{0\}$, let $\pi_{I}$ be the projection of $\mathbb{N}^{m}$ onto $\prod_{i \in I} M_{i}$, and let $\chi^{\prime}$ be the unique natural embedding satisfying $\chi(v)=\chi^{\prime}\left(\pi_{I}(v)\right)$, for all $v \in \mathbb{N}^{m}$, as defined at the beginning of this section. Then, we get

$$
\chi\left(X \cap M_{1} \times \cdots \times M_{m}\right)=\chi^{\prime}\left(\pi_{I}(X) \cap \prod_{i \in I} M_{i}\right) .
$$

Whenever $I=\emptyset$, we have $\chi\left(X \cap M_{1} \times \cdots \times M_{m}\right)=\{\varepsilon\}$.

\subsection{The Letter Case}

A morphism of a free monoid into another is nonincreasing if the image of a letter is a letter or the empty word. A length-preserving substitution is defined by a mapping $h: \Sigma \rightarrow$ $2^{\Sigma} \backslash\{\emptyset\}$, and assigns to the word $a_{1} \cdots a_{n}$ the set of words $h\left(a_{1}\right) \cdot \ldots \cdot h\left(a_{n}\right)$. It extends to subsets of words in the usual way.

Proposition 1 If $L, L^{\prime} \subseteq a_{1}^{*} \cdots a_{m}^{*}$ are letter bounded semilinear languages and if $f$ is a nonincreasing morphism, then $L \cup L^{\prime}, L \backslash L^{\prime}, L L^{\prime}$, and $f(L)$ are letter bounded semilinear. Furthermore, if $h$ is a length-preserving substitution, then $L \cap h\left(L^{\prime}\right)$ is letter bounded semilinear.

Proof The first three properties are consequences of the results in [8]. Concerning nonincreasing morphisms, observe that they are a composition of morphisms of two types: those renaming a letter and leaving all other letters invariant and those sending a letter to the empty word and leaving all other letters invariant. In the former case, the result follows directly from the above observation concerning the non-repetition of two consecutive words. In the latter case, assume the morphism $f$ satisfies $f(a)=\varepsilon$ and $f(c)=c$ for all $c \in \Sigma \backslash\{a\}$. Let $I$ the subset of indices $i \in\{1, \ldots, m\}$ such that $a_{i}=a$, and denote by $\pi$ the morphism of $\mathbb{N}^{m}$ into $\mathbb{N}^{m-|I|}$ assigning to any $m$-tuple the $(m-|I|)$-tuple obtained by ignoring the components whose positions are in $I$. If $L=\chi(X)$ then $f(L)=\chi^{\prime}(\pi(X))$, where $\chi^{\prime}$ is the obvious restriction of $\chi$, and we apply the closure property under morphism of the semilinear sets [8].

The last statement follows from the fact that the intersection of a bounded semilinear language with the image of a bounded semilinear language under a substitution by regular sets is bounded semilinear [11, Thm 5.5]. Observe that $h\left(L^{\prime}\right)$ is not bounded in general, which is why we consider the intersection with a bounded language.

We are now going to show that every letter bounded language is in $\mathscr{L}(\mathrm{FO}[+])$ if and only if it is semilinear. We start with the "if" part.

Theorem 1 The class of letter bounded semilinear languages is in $\mathscr{L}(\mathrm{FO}[+])$.

Proof It clearly suffices to prove the result for letter bounded linear languages, i.e., languages of the form $L=\chi(X)$ where $X \subseteq \mathbb{N}^{m}$ is linear and $\chi$ is the natural embedding of $\mathbb{N}^{m}$ into $a_{1}^{*} \cdots a_{m}^{*}$, for some sequence $a_{1}, \ldots, a_{m}$ of letters from $\Sigma$. 
By Lemma 1, we may assume that $L \subseteq a_{1}^{+} \cdots a_{m}^{+}$. We give an $\mathrm{FO}[+]$ formula defining the letter bounded linear language $L=\chi(X)$, with $X=v_{0}+\sum_{t=1}^{r} \mathbb{N} v_{t}$. By definition, a word $w \in \Sigma^{*}$ belongs to $L$ if and only if:

(i) $w$ is in $a_{1}^{+} \cdots a_{m}^{+}$, and

(ii) for some $\alpha_{1}, \ldots, \alpha_{r} \in \mathbb{N}$, it holds $|w|_{a_{j}}=v_{0, j}+\sum_{t=1}^{r} \alpha_{t} v_{t, j}$ for $1 \leq j \leq m$, where $v_{t, j}$ denotes the $j$ th component of the vector $v_{t}$.

Let the variables $y_{1}, \ldots, y_{m}$ be interpreted as the number of occurrences of the letters $a_{1}, \ldots, a_{m}$ in $w$. Then, an $\mathrm{FO}[+]$ formula defining $L$ is of the form

$$
\exists y_{1} \ldots \exists y_{m} \psi_{1}\left(y_{1}, \ldots, y_{m}\right) \wedge \psi_{2}\left(y_{1}, \ldots, y_{m}\right),
$$

where $\psi_{1}$ expresses condition (i) and $\psi_{2}$ expresses condition (ii). Using natural abbreviations in order to keep the formula readable, we have

$$
\begin{aligned}
& \psi_{1}\left(y_{1}, \ldots, y_{m}\right) \equiv\left(y_{1}+\cdots+y_{m}=\text { last }\right) \wedge \\
& \forall z\left(\left(z \leq y_{1} \Rightarrow Q_{a_{1}}(z)\right) \wedge \bigwedge_{i=2}^{m}\left(\sum_{h=1}^{i-1} y_{h}<z \leq \sum_{h=1}^{i} y_{h} \Rightarrow Q_{a_{i}}(z)\right)\right) .
\end{aligned}
$$

Concerning condition (ii), by denoting with $z_{j}$ the variables interpreted as the coefficients $\alpha_{j}$, we have

$$
\psi_{2}\left(y_{1}, \ldots, y_{m}\right) \equiv \exists z_{1} \cdots \exists z_{r}\left(\bigwedge_{1 \leq j \leq m}\left(y_{j}=v_{0, j}+\sum_{t=1}^{r} \sum_{s=1}^{v_{t, j}} z_{t}\right)\right)
$$

The converse of Theorem 1 goes by structural induction on $\mathrm{FO}[+]$ formulas. Indeed, we consider not only sentences (i.e., formulas with only bound variables) but more generally formulas with free variables, and define what it means for such formulas to be satisfied by some model. We utilize the usual trick which consists of augmenting the letters of the alphabet $\Sigma$ with a new component specifying subsets of free variables: by so doing, we encode the value of the free variables in the model. More precisely, a formula $\phi$ over a set $\mathscr{V}$ of free variables is interpreted on $\mathscr{V}$-structures, i.e., words of the form $u=\left(\sigma_{1}, \mathscr{V}_{1}\right) \cdots\left(\sigma_{n}, \mathscr{V}_{n}\right)$ over the alphabet $\Sigma \times 2^{\mathscr{V}}$, with: (i) $\mathscr{V}_{i} \subseteq \mathscr{V}$, (ii) $\mathscr{V}_{i} \cap \mathscr{V}_{j}=\emptyset$ for $i \neq j$, (iii) $\bigcup_{i=1}^{n} \mathscr{V}_{i}=\mathscr{V}$. We let $\mathscr{S}_{|\mathscr{V}|} \subseteq\left(\Sigma \times 2^{\mathscr{V}}\right)^{*}$ denote the set of all $\mathscr{V}$-structures.

Let us now explain what it means for a $\mathscr{V}$-structure to satisfy a formula with free variables (the figure below should facilitate the intuition). To fix ideas, let $\phi\left(x_{1}, \ldots, x_{k}\right)$ be a formula and $\mathscr{V}=\left\{x_{1}, \ldots, x_{k}\right\}$ be the set of its free variables. We say that the $\mathscr{V}$-structure $u=\left(\sigma_{1}, \mathscr{V}_{1}\right) \cdots\left(\sigma_{n}, \mathscr{V}_{n}\right) \in \mathscr{S}_{|\mathscr{V}|}$ satisfies $\phi\left(x_{1}, \ldots, x_{k}\right)$, and we write $u=\phi\left(x_{1}, \ldots, x_{k}\right)$, if $\phi\left(p_{1}, \ldots, p_{k}\right)$ holds true in the model $\sigma_{1} \cdots \sigma_{n}$ where, for $1 \leq i \leq k$, the integer $1 \leq p_{i} \leq n$ is the unique position of the $\mathscr{V}$-structure $u$ such that $x_{i} \in \mathscr{V}_{p_{i}}$. For instance, the following $\mathscr{V}$-structure

\begin{tabular}{|c|c|c|c|c|c|}
\hline$a$ & $b$ & $a$ & $a$ & $b$ & $b$ \\
\hline$\emptyset$ & $\left\{x_{2}\right\}$ & $\left\{x_{3}, x_{4}\right\}$ & $\emptyset$ & $\left\{x_{1}\right\}$ & $\emptyset$ \\
\hline
\end{tabular}

satisfies the formula

$$
Q_{b}\left(x_{1}\right) \wedge Q_{b}\left(x_{2}\right) \wedge \neg Q_{b}\left(x_{3}\right) \wedge\left(x_{2}<x_{3}\right) \wedge\left(x_{3}<x_{1}\right) \wedge\left(x_{2}<x_{4}\right) \wedge\left(x_{4}<x_{1}\right) .
$$

The language defined by $\phi$ is the set $L_{\phi, \mathscr{V}}=\left\{u \in \mathscr{S}_{|\mathscr{V}|}|u|=\phi\right\}$. If $\phi$ is a sentence, i.e., a formula without free variables, then $L_{\phi}=L_{\phi, \emptyset}=\left\{w \in \Sigma^{*}|w|=\phi\right\}$, as recalled in Section 2 . 
The following lemma, which is useful in the proof of the main result, shows the letter boundedness and semilinearity of a language of $\mathscr{V}$-structures. Given a $\mathscr{V}$-structure $u=$ $\left(\sigma_{1}, \mathscr{V}_{1}\right) \cdots\left(\sigma_{n}, \mathscr{V}_{n}\right)$, we define $\pi(u)=\sigma_{1} \cdots \sigma_{n}$. Then:

Lemma 2 Let $a_{1}, \ldots, a_{m}$ be a sequence of letters from an alphabet $\Sigma$ and let $\mathscr{V}$ be a set of free variables with $|\mathscr{V}|=k$. The language

$$
B_{k}=\left\{u \in \mathscr{S}_{k} \mid \pi(u) \in a_{1}^{*} \cdots a_{m}^{*}\right\}
$$

is letter bounded semilinear.

Proof The set $a_{1}^{*} \cdots a_{m}^{*}$ is the (finite) union of subsets of the form $a_{i_{1}}^{+} \cdots a_{i_{s}}^{+}$where $0 \leq s \leq m$ and $i_{1}, \ldots, i_{s}$ is a subsequence of $1, \ldots, m$. So, we are reduced to prove that the set $B_{k}^{\prime}=$ $\left\{u \in \mathscr{S}_{k} \mid \pi(u) \in a_{1}^{+} \cdots a_{m}^{+}\right\}$is letter bounded semilinear.

We claim that $B_{k}^{\prime}$ is a finite union of letter bounded linear languages. A subset in this union is specified by the choice of a sequence of nonempty subsets $\mathscr{V}_{1}, \ldots, \mathscr{V}_{\ell}$ defining a decomposition of $\mathscr{V}$ and a choice of letters from $a_{1}, \ldots, a_{m}$ which are associated with the $\mathscr{V}_{i}$ 's. For instance, let $k=3$ and $m=3$, i.e., we are considering the words in $a_{1}^{+} a_{2}^{+} a_{3}^{+}$and the formula has 3 free variables $x_{1}, x_{3}, x_{3}$. Consider the decomposition $\mathscr{V}=\mathscr{V}_{1} \cup \mathscr{V} / 2$ with $\mathscr{V}_{1}=\left\{x_{1}, x_{3}\right\}$ and $\mathscr{V}_{2}=\left\{x_{2}\right\}$. Associate $\mathscr{V}_{1}$ with $a_{1}$ and $\mathscr{V}_{2}$ with $a_{3}$. Then, the associated subset is

$$
\left(a_{1}, \emptyset\right)^{*}\left(a_{1}, \mathscr{V}_{1}\right)\left(a_{1}, \emptyset\right)^{*}\left(a_{2}, \emptyset\right)^{*}\left(a_{3}, \emptyset\right)^{*}\left(a_{3}, \mathscr{V}_{2}\right)\left(a_{3}, \emptyset\right)^{*},
$$

which indicates that the positions of the interpretations of the variables $x_{1}$ and $x_{3}$ are inside the factor of the word labeled by $a_{1}$, while the interpretation of the variable $x_{2}$ is inside the factor of the word labeled by $a_{3}$.

Formally, consider a sequence of the form $\left(i_{1}, \mathscr{V}_{1}\right), \ldots,\left(i_{\ell}, \mathscr{V}_{\ell}\right)$ satisfying the following conditions:

$-1 \leq i_{1}<\cdots<i_{\ell} \leq m$,

- $\mathscr{V}_{i_{\alpha}} \neq \emptyset$, for $1 \leq \alpha \leq \ell$,

- $\bigcup_{\alpha=1}^{\ell} \mathscr{V}_{i_{\alpha}}=\mathscr{V}$ and $\mathscr{V}_{i_{\alpha}} \cap \mathscr{V}_{i_{\beta}}=\emptyset$, for $1 \leq \alpha<\beta \leq \ell$.

Set $i_{0}=1$ and $i_{\ell+1}=m$, and define

$$
L_{\alpha}=\left(a_{i_{\alpha-1}}, \emptyset\right)^{*} \cdots\left(a_{i_{\alpha}}, \emptyset\right)^{*}\left(a_{i_{\alpha}}, \mathscr{V}_{\alpha}\right)\left(a_{i_{\alpha}}, \emptyset\right)^{*} \cdots\left(a_{i_{\alpha+1}}, \emptyset\right)^{*} .
$$

Then, each of these $L_{\alpha}$ is letter bounded linear and thus, by Proposition 1, the product $L_{1} \cdots L_{\ell}$ is letter bounded linear as well. Since $B_{k}^{\prime}$ is a finite union of such languages, it is letter bounded semilinear.

Hereafter, for the sake of conciseness, given an alphabet $\Sigma$ we let $Q_{\Sigma}(x)$ stand for $\bigvee_{\sigma \in \Sigma} Q_{\sigma}(x)$. We obtain the converse of Theorem 1 as a corollary of

Theorem 2 For every sequence $a_{1}, \ldots, a_{m}$ of letters in $\Sigma$ and every language $L \subseteq \Sigma^{*}$ in $\mathscr{L}(\mathrm{FO}[+])$, the language $L \cap a_{1}^{*} \cdots a_{m}^{*}$ is semilinear.

Proof Let $\phi$ be an $\mathrm{FO}[+]$ formula with $\mathscr{V}=\left\{x_{1}, \ldots, x_{k}\right\}$ free variables. To prove the result, it is enough to show that $L_{\phi, \mathscr{V}} \cap B_{k}$ is semilinear, where $B_{k}=\left\{u \in \mathscr{S}_{k} \mid \pi(u) \in a_{1}^{*} \cdots a_{m}^{*}\right\}$ is the letter bounded semilinear language in Lemma 2. We shall use the structural induction on $\phi$, starting from atomic predicates and then consider more complex formulas. 
- $\phi \equiv Q_{a}(x)$ : If $a$ does not occur in the sequence $a_{1}, \ldots, a_{m}$, then $L_{\phi, \mathscr{V}} \cap B_{1}=\emptyset$, so we assume $a=a_{i}$ for some $1 \leq i \leq m$. We have

$$
L_{\phi,\{x\}} \cap B_{1}=\left(a_{1}, \emptyset\right)^{*} \cdots\left(a_{i}, \emptyset\right)^{*}\left(a_{i},\{x\}\right)\left(a_{i}, \emptyset\right)^{*} \cdots\left(a_{m}, \emptyset\right)^{*},
$$

which is clearly letter bounded linear.

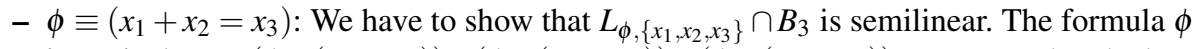
is equivalent to $\left(\phi \wedge\left(x_{1}<x_{2}\right)\right) \vee\left(\phi \wedge\left(x_{1}=x_{2}\right)\right) \vee\left(\phi \wedge\left(x_{1}>x_{2}\right)\right)$. We prove that the language $L_{\phi_{<},\left\{x_{1}, x_{2}, x_{3}\right\}} \cap B_{3}$ with $\phi_{<} \equiv \phi \wedge\left(x_{1}<x_{2}\right)$ is letter bounded linear. The two other cases are treated similarly. For $W \subseteq\left\{x_{1}, x_{2}, x_{3}\right\}$, let $A_{W}=\left\{(\sigma, W) \mid \sigma \in\left\{a_{1}, a_{2}, \ldots, a_{m}\right\}\right\}$. Then

$$
L_{\phi_{<},\left\{x_{1}, x_{2}, x_{3}\right\}}=\left\{A_{\emptyset}{ }^{\alpha} A_{\left\{x_{1}\right\}} A_{\emptyset}{ }^{\beta} A_{\left\{x_{2}\right\}} A_{\emptyset}{ }^{\alpha} A_{\left\{x_{3}\right\}} A_{\emptyset}{ }^{\gamma} \mid \alpha, \beta, \gamma \in \mathbb{N}\right\} .
$$

This language is the image under the length-preserving substitution defined by $h(a)=$ $A_{\emptyset}, h(b)=A_{\left\{x_{1}\right\}}, h(c)=A_{\left\{x_{2}\right\}}$ and $h(d)=A_{\left\{x_{3}\right\}}$ of the letter bounded linear language $L^{\prime}=\left\{a^{\alpha} b a^{\beta} c a^{\alpha} d^{\gamma} \mid \alpha, \beta, \gamma \in \mathbb{N}\right\}$. So

$$
L_{\phi_{<},\left\{x_{1}, x_{2}, x_{3}\right\}} \cap B_{3}=h\left(L^{\prime}\right) \cap B_{3},
$$

and the result follows from Lemma 2 and Proposition 1.

$-\phi \equiv \neg \psi:$ We have

$$
\begin{aligned}
L_{\phi, \mathscr{V}} \cap B_{k} & =L_{\neg \psi, \mathscr{V}} \cap B_{k}=\left(\mathscr{S}_{k} \backslash L_{\psi, \mathscr{V}}\right) \cap B_{k}=\left(\mathscr{S}_{k} \cap L_{\psi, \mathscr{V}}^{c}\right) \cap B_{k} \\
& =L_{\psi, \mathscr{V}}^{c} \cap B_{k}=B_{k} \cap\left(L_{\psi, \mathscr{V}}^{c} \cup B_{k}^{c}\right)=B_{k} \backslash\left(L_{\psi, \mathscr{V}} \cap B_{k}\right) .
\end{aligned}
$$

By inductive hypothesis, we have that $L_{\psi, \mathscr{V}} \cap B_{k}$ is letter bounded semilinear. The result follows from Lemma 2 and Proposition 1.

- $\phi \equiv \psi_{1} \wedge \psi_{2}$ : We first transform $\psi_{1}$ and $\psi_{2}$ into equivalent formulas, each over the same set of free variables, say $\mathscr{V}$. To this purpose, let $\mathscr{W}_{1}$ and $\mathscr{W}_{2}$ be the set of free variables of $\psi_{1}$ and $\psi_{2}$, respectively, so that $\mathscr{V}=\mathscr{W}_{1} \cup \mathscr{W}_{2}$. Define $\hat{\psi}_{1} \equiv \psi_{1} \wedge \bigwedge_{x \in \mathscr{N} \backslash \mathscr{W}_{1}} Q_{\Sigma}(x)$ and $\hat{\psi}_{2} \equiv \psi_{2} \wedge \bigwedge_{x \in \mathscr{N} \backslash \mathscr{W}_{2}} Q_{\Sigma}(x)$. Clearly, $\phi$ is equivalent to $\hat{\psi}_{1} \wedge \hat{\psi}_{2}$. We have

$$
\begin{aligned}
& L_{\phi, \mathscr{V}} \cap B_{k}=L_{\hat{\psi}_{1} \wedge \hat{\psi}_{2}, \mathscr{V}} \cap B_{k}=L_{\hat{\psi}_{1}, \mathscr{V}} \cap L_{\hat{\psi}_{2}, \mathscr{V}} \cap B_{k} \\
& =\left(L_{\hat{\psi}_{1}, \mathscr{V}} \cap B_{k}\right) \cap\left(L_{\hat{\psi}_{2}, \mathscr{V}} \cap B_{k}\right) \text {. }
\end{aligned}
$$

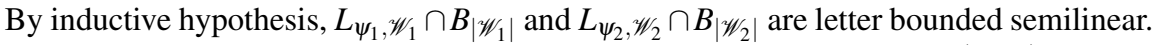
Let $h$ be the length-preserving substitution which assigns to every letter $(a, W) \in \mathscr{S}_{\left|W_{1}\right|}$ the set of letters $(a, V) \in \mathscr{S}_{k}$ where $V=W \cup A$, for $A \subseteq \mathscr{W}_{2} \backslash \mathscr{W}_{1}$. Then, we have

$$
L_{\hat{\psi}_{1}, \mathscr{V}} \cap B_{k}=h\left(L_{\psi_{1}, \mathscr{W}_{1}} \cap B_{\left|\mathscr{W}_{1}\right|}\right) \cap B_{k}
$$

The result follows from Lemma 2 and Proposition 1.

- $\phi \equiv \exists x_{k+1} \psi\left(x_{1}, \ldots, x_{k}, x_{k+1}\right)$ : Let the nonincreasing morphism $\Pi: \mathscr{S}_{k+1} \rightarrow \mathscr{S}_{k}$ be defined as $\Pi(a, V)=\left(a, V \backslash\left\{x_{k+1}\right\}\right)$. E.g., if $u=(a, \emptyset)\left(a,\left\{x_{3}\right\}\right)\left(b,\left\{x_{1}\right\}\right)\left(b,\left\{x_{2}\right\}\right) \in \mathscr{S}_{3}$, we have $\Pi(u)=(a, \emptyset)(a, \emptyset)\left(b,\left\{x_{1}\right\}\right)\left(b,\left\{x_{2}\right\}\right) \in \mathscr{S}_{2}$. We extend $\Pi$ to subsets of $\mathscr{S}_{k+1}$ in the usual way. Notice that $B_{k}=\Pi\left(B_{k+1}\right)$. We have

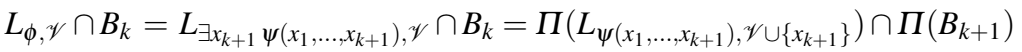

$$
\begin{aligned}
& =\Pi\left(L_{\psi\left(x_{1}, \ldots, x_{k+1}\right), \mathscr{V} \cup\left\{x_{k+1}\right\}} \cap B_{k+1}\right) .
\end{aligned}
$$

The last equality follows from the saturation of $B_{k+1}$ relative to $\Pi$, i.e., from the fact that $\Pi(u)=\Pi(v)$ and $u \in B_{k+1}$ implies $v \in B_{k+1}$. By inductive hypothesis, we have that $L_{\psi\left(x_{1}, \ldots, x_{k+1}\right), \mathscr{V} \cup\left\{x_{k+1}\right\}} \cap B_{k+1}$ is letter bounded semilinear, and the result follows from Proposition 1. 
In conclusion, from Theorems 1 and 2, we get

Theorem 3 A letter bounded language is semilinear if and only if it belongs to $\mathscr{L}(\mathrm{FO}[+])$.

\subsection{From Letters to Words}

We show how to extend the result from letter bounded to word bounded languages. From now on, the term bounded means word bounded unless otherwise stated.

Theorem 4 Let $L$ be a bounded language. Then, $L$ is semilinear if and only if $L$ belongs to $\mathscr{L}(\mathrm{FO}[+])$.

Proof By Lemma 1, we assume without loss of generality that the semilinear bounded language we start with satisfies the further condition

$$
L=\left\{w_{1}^{r_{1}} \cdots w_{m}^{r_{m}} \mid\left(r_{1}, \ldots, r_{m}\right) \in X\right\}
$$

where $X$ consists of $m$-tuples with nonzero components. We also introduce the alphabet $\left\{a_{1}, \ldots, a_{m}\right\}$ of $m$ new distinct symbols, where $a_{i}$ is in correspondence with $w_{i}$. Observe that there might be several occurrences of the same word. We construct an $\mathrm{FO}[+]$ formula which defines $L$. Denoting by $Y$ the componentwise product $\left(\left|w_{1}\right|, \ldots,\left|w_{m}\right|\right) X$, the subset

$$
L^{\prime}=\left\{a_{1}^{\left|w_{1}\right| r_{1}} \ldots a_{m}^{\left|w_{m}\right| r_{m}} \mid\left(\left|w_{1}\right| r_{1}, \ldots,\left|w_{m}\right| r_{m}\right) \in Y\right\}
$$

is linear and therefore there exists an $\mathrm{FO}[+]$ formula $\phi^{\prime}$ for $L^{\prime}$. Let us introduce the following predicates:

- $\psi_{a_{i}}\left(x, y, x^{\prime}\right) \equiv x \leq y \leq x^{\prime}, i=1, \ldots, m$.

- $\gamma_{i}\left(x, x^{\prime}\right)$ which holds true whenever the factor of a word between the position $x$ and $x^{\prime}$, both ends included, belongs to $w_{i}^{+}$. Formally, set $w_{i}=w_{i, 1} \ldots w_{i, k_{i}}$. We define

$$
\begin{aligned}
& \gamma_{i}\left(x, x^{\prime}\right) \equiv \bigwedge_{1 \leq j \leq k_{i}} Q_{w_{i, j}}(x-1+j) \wedge \\
& \forall y\left(\left(x \leq y \leq x^{\prime}-k_{i}\right) \wedge\left(\bigwedge_{1 \leq j \leq k_{i}} Q_{w_{i, j}}(y) \Rightarrow \bigwedge_{1 \leq j \leq k_{i}} Q_{w_{i, j}}\left(y+k_{i}\right)\right)\right) .
\end{aligned}
$$

Then, the $\mathrm{FO}[+]$ formula for $L$ is as follows:

$$
\exists x_{1} \cdots \exists x_{m+1}\left(\left(1=x_{1}<\cdots<x_{m+1}=\text { last }\right) \wedge \gamma_{1}\left(x_{1}, x_{2}\right) \wedge \bigwedge_{1<i \leq m} \gamma_{i}\left(x_{i}+1, x_{i+1}\right) \wedge \phi\right),
$$

where $\phi$ is obtained from $\phi^{\prime}$ by substituting $\psi_{a_{1}}\left(x_{1}, y, x_{2}\right)$ for $Q_{a_{1}}(y)$ and, for $1<i \leq m$, $\psi_{a_{i}}\left(x_{i}+1, y, x_{i+1}\right)$ for $Q_{a_{i}}(y)$.

Conversely, consider a bounded language $L$ which is defined by the formula $\phi$ in $\mathrm{FO}[+]$. The idea is to transform every word $w_{1}^{r_{1}} \cdots w_{m}^{r_{m}}$ into $a_{1}^{\left|w_{1}\right| r_{1}} \cdots a_{m}^{\left|w_{m}\right| r_{m}}$ and to show that the subset $L^{\prime}$ of $a_{1}^{*} \cdots a_{m}^{*}$ thus obtained is definable in $\mathrm{FO}[+]$. Without loss of generality, here we consider the case $r_{1}, \ldots, r_{m}>0$ :

$$
\begin{aligned}
& \exists x_{1} \cdots \exists x_{m+1}\left(\left(1=x_{1}<\cdots<x_{m+1}=\text { last }\right) \wedge \forall y\left(x_{1} \leq y \leq x_{2} \Rightarrow Q_{a_{1}}(y)\right) \wedge\right. \\
& \left.\left(\bigwedge_{1<i \leq m} \forall y\left(x_{i}<y \leq x_{i+1} \Rightarrow Q_{a_{i}}(y)\right)\right) \wedge \phi^{\prime}\right),
\end{aligned}
$$


where the formula $\phi^{\prime}$ is obtained from the formula $\phi$ by substituting every occurrence of $Q_{\sigma}(y)$ by the following predicate. Let $I \subseteq\{1, \ldots, m\}$ be such that $i \in I$ implies that $w_{i}$ contains the symbol $\sigma$, and let $\left|w_{i}\right|=k_{i}$. Then:

$$
\psi_{\sigma}(y) \equiv \bigvee_{i \in I} \bigvee_{w_{i, j}=\sigma} \exists z\left(y=x_{i}+1+j+k_{i} z\right)
$$

By Theorem 3, the subset $L^{\prime}$ is semilinear, therefore so is the subset $L$ as can be readily verified. This completes the proof.

\section{Closure Properties}

In this section, we investigate the closure properties of the classes $\mathscr{L}(\mathrm{FO}[+1]), \mathscr{L}(\mathrm{FO}[<])$, $\mathscr{L}(\mathrm{FO}[+])$ and $\mathscr{L}(\mathrm{FOC}[+])$ under various operations. The results of the previous section help us to show some negative closure results. On the other hand, also some positive closure results are obtained. The complete picture is displayed in Figure 1 of the Introduction.

The following notation will be used in this section: Let $\phi\left(x_{1}, \ldots, x_{r}\right)$ be a formula with $x_{1}, \ldots, x_{r}$ free variables. We write $\left(w, a_{1}, \ldots, a_{r}\right) \models \phi$ whenever $w \mid=\phi\left(a_{1}, \ldots, a_{r}\right)$.

\subsection{Morphisms}

We need a more precise classification of the morphisms. We recall that a morphism $h: \Sigma^{*} \rightarrow$ $\Delta^{*}$ of a free monoid into another is entirely defined by the image of each letter of $\Sigma$. It is an elementary result that $h$ can be expressed as a composition of morphisms $g$ of one of the following types:

- $g$ defines a permutation on the symbols of $\Sigma$ and is called permutation morphism.

- $g$ identifies two letters and leaves all other letters invariant, i.e., there exist $a, b \in \Sigma$ such that $g(a)=g(b)=a$ and $g(c)=c$ for all $c \neq a, b$. Call this morphism identifying.

- $g$ deletes some letter and leaves all other letters unchanged, i.e, there exists $a \in \Sigma$ such that $g(a)=\varepsilon$ and $g(b)=b$ for all $b \neq a$. This morphism is called erasing.

- there exists a letter $a \in \Sigma$ such that $g(a)=a c$ and $g(b)=b$ for all $b \neq a$. Let us call the morphism growing.

Consistently with the definition given at the beginning of Section 3.2, we say that a morphism $h: \Sigma^{*} \rightarrow \Delta^{*}$ is length-preserving whenever $|h(w)|=|w|$, for every $w \in \Sigma^{*}$. We start by showing that none of the classes considered are closed under length-preserving homomorphisms.

Proposition 2 The classes $\mathscr{L}(\mathrm{FO}[+1]), \mathscr{L}(\mathrm{FO}[<]), \mathscr{L}(\mathrm{FO}[+])$ and $\mathscr{L}(\mathrm{FOC}[+])$ are not closed under length-preserving homomorphism.

Proof Consider $\Sigma=\{a, b\}$ and the language $L=(a b)^{+}$. Notice that $(a b)^{+}=a \Sigma^{*} \cap \Sigma^{*} b \backslash$ $\Sigma^{*}\left\{a^{2}, b^{2}\right\} \Sigma^{*}$ holds, which implies $L$ is a local set and belongs to $\mathscr{L}(\mathrm{FO}[+1])$ and thus to $\mathscr{L}(\mathrm{FO}[<])$. Now, consider the length-preserving homomorphism $h:\{a, b\}^{+} \rightarrow\{a\}^{+}$ defined as $h(a)=h(b)=a$. We obtain $h(L)=\left(a^{2}\right)^{+}$, which does not belong to $\mathscr{L}(\mathrm{FO}[<])$.

Concerning $\mathscr{L}(\mathrm{FO}[+])$, we start by showing that the language

$$
L=\left\{a b a b^{2} a b^{3} a \cdots a b^{i} a \cdots a b^{k} a \mid k \in \mathbb{N} \backslash\{0\}\right\}
$$


belongs to $\mathscr{L}(\mathrm{FO}[+])$ but $h(L)$ does not belong to $\mathscr{L}(\mathrm{FOC}[+])$, where $h$ is the lengthpreserving homomorphism above defined. Observe that $w \in\{a, b\}^{*}$ belongs to $L$ if and only if both the following conditions hold true:

- $w=a b a$ or $w \in a b a \Sigma^{*} a$,

- if $w \neq a b a$ and $w=u a b^{i} a v$, with $u, v \in\{a, b\}^{*}$ and $i>0$, then there exists a suffix $v^{\prime}$ of $v$ such that $v=b^{i+1} a v^{\prime}$.

These two conditions can be expressed by the following $\mathrm{FO}[+]$ formula

$$
\begin{aligned}
& Q_{a}(1) \wedge Q_{b}(2) \wedge Q_{a}(3) \wedge Q_{a}(\text { last }) \wedge \\
& \quad \forall x\left[\left(\exists y \exists z Q_{a}(x) \wedge Q_{a}(x+y) \wedge Q_{a}(x+y+z)\right) \Rightarrow(\exists y \phi(x, y))\right],
\end{aligned}
$$

with

$$
\begin{aligned}
\phi(x, y) \equiv & Q_{a}(x) \wedge Q_{a}(x+y+1) \wedge Q_{a}(x+2 y+3) \wedge \\
& \forall z\left(x<z<x+y+1 \Rightarrow Q_{b}(z)\right) \wedge \\
& \forall z\left(x+y+1<z<x+2 y+3 \Rightarrow Q_{b}(z)\right) .
\end{aligned}
$$

Now, we have

$$
h(L)=\left\{a^{\frac{(p+1)(p+2)}{2}} \mid p \in \mathbb{N} \backslash\{0\}\right\} .
$$

By Theorem 2, $h(L) \notin \mathscr{L}(\mathrm{FO}[+])$. Moreover, we know from [17] that $\mathrm{FO}[+]$ and $\mathrm{FOC}[+]$ have the same expressive power when used for unary languages. This implies that $h(L) \notin$ $\mathscr{L}(\mathrm{FOC}[+])$ as well.

The situation is slightly different concerning the inverse morphisms.

Proposition 3 The class $\mathscr{L}(\mathrm{FO}[<])$ is closed under inverse homomorphism, but the classes $\mathscr{L}(\mathrm{FO}[+1])$ and $\mathscr{L}(\mathrm{FO}[+])$ are not. All three classes are closed under inverse lengthpreserving homomorphism.

Proof The assertion for $\mathscr{L}(\mathrm{FO}[<])$ follows from the fact that it is a pseudovariety of languages [18]. Concerning the nonclosure of $\mathscr{L}(\mathrm{FO}[+1])$ and $\mathscr{L}(\mathrm{FO}[+])$, we observe that $X=\{a b\} \in \mathscr{L}(\mathrm{FO}[+1])$ and $Y=\left\{\left(a^{2}\right)^{*}\right\} \in \mathscr{L}(\mathrm{FO}[+]) \backslash \mathscr{L}(\mathrm{FO}[<])$. Consider the morphisms $h:\{a, b, c\}^{*} \rightarrow\{a, b\}^{*}$ and $g:\{a, b\}^{*} \rightarrow\{a\}^{*}$ defined by $h(a)=a, h(b)=b, h(c)=\varepsilon$ and $g(a)=a, g(b)=\varepsilon$. Then, $h^{-1}(X)=c^{*} a c^{*} b c^{*} \notin \mathscr{L}(\mathrm{FO}[+1])$ and, by [2, Cor. 4.2] which is the Crane-Beach conjecture for $\mathrm{FO}[+], g^{-1}(Y)=\left\{\left.w \in\{a, b\}^{+}|| w\right|_{a} \bmod 2=0\right\}$ $\notin \mathscr{L}(\mathrm{FO}[+])$.

The last statement holds in all generality. Indeed, a length-preserving homomorphism $h$ is a composition of permutation morphisms and identifying morphisms. We thus only consider the latter case. Let $a, b \in \Sigma, h(a)=h(b)=a$ and $h(c)=c$ whenever $c \neq a, b$. If $X=L_{\phi}$ then $h^{-1}(X)=L_{\phi^{\prime}}$ where $\phi^{\prime}$ is obtained from $\phi$ by substituting $Q_{a}(x) \vee Q_{b}(x)$ for each occurrence of $Q_{a}(x)$.

The class FOC $[+]$ behaves very differently since it is closed under all inverse homomorphisms. We need a preliminary general result showing that under general conditions and provided the language allows counting quantifiers, it is possible to simulate a structure into another. This idea is similar to the notion of FO-reduction as exposed, e.g., in [6]. We prove that in this embedding, sums and counting quantifications in the first structure are expressible in the other. Given a total ordering, we call rank of an element $x$ the number $\rho(x)$ of elements less than or equal to $x$, i.e., $\rho(x)=|\{y \mid y \leq x\}|$. 
Proposition 4 Let $U(x, y) \in \mathrm{FOC}[+]$ be a predicate, let $\left(x_{1}, y_{1}\right) \leq\left(x_{2}, y_{2}\right)$ be a predicate in FOC $[+]$ which defines a total ordering on $\{(x, y) \mid U(x, y)\}$ and let $\rho(x, y)$ be the rank of $(x, y)$ in this ordering. Let $k$ be an integer such that $(a, b) \models U(x, y)$ implies $a \leq k$. Then:

(i) There exists a formula $\theta\left(x_{1}, y_{1}, x_{2}, y_{2}, x_{3}, y_{3}\right)$ in $\mathrm{FOC}[+]$ which holds true if and only if for $\left(x_{1}, y_{1}\right),\left(x_{2}, y_{2}\right),\left(x_{3}, y_{3}\right) \models U(x, y)$ the following property holds:

$$
\rho\left(x_{1}, y_{1}\right)+\rho\left(x_{2}, y_{2}\right)=\rho\left(x_{3}, y_{3}\right) \text {. }
$$

(ii) Let $\phi\left(\left(x_{1}, y_{1}\right), \ldots,\left(x_{p}, y_{p}\right)\right)$ be an FOC $[+]$ formula holding true only when $\left(x_{i}, y_{i}\right) \models$ $U(x, y)$ for $i=1, \ldots, p$. Then, there exists an $\mathrm{FOC}[+]$ formula

$$
\psi\left((x, y),\left(x_{2}, y_{2}\right), \ldots,\left(x_{p}, y_{p}\right)\right)
$$

which holds true for all assignments $\left(a_{2}, b_{2}\right), \ldots,\left(a_{p}, b_{p}\right)$ of $\left(x_{2}, y_{2}\right), \ldots,\left(x_{p}, y_{p}\right)$ exactly for the value $(a, b)$ of $(x, y)$, if it exists, for which

$$
\rho(a, b)=\left|\left\{\left(a_{1}, b_{1}\right) \mid \phi\left(\left(a_{1}, b_{1}\right), \cdots,\left(a_{p}, b_{p}\right)\right)\right\}\right|
$$

holds true.

Proof We may not directly define values which exceed last. So we will first explain how to manage values exceeding last, by representing ranks and differences of ranks less than or equal to last.

We explain intuitively how we proceed. Equality (1) is equivalent to

$$
\rho\left(x_{2}, y_{2}\right)=\rho\left(x_{3}, y_{3}\right)-\rho\left(x_{1}, y_{1}\right) \text {. }
$$

In order to express it, we consider the graph of the total ordering enriched with a dummy element of rank 0 . The value $\rho\left(x_{3}, y_{3}\right)-\rho\left(x_{1}, y_{1}\right)$ is less than $k$ - last, which means that there exists a path of length less than or equal to $k$, starting from $\left(x_{1}, y_{1}\right)$ and ending in $\left(x_{3}, y_{3}\right)$ such that the difference of the ranks of two successive elements is less than or equal to last. Equality (3) can thus be expressed by stating that there exists a path of the same length starting with the element of rank 0 , ending in $\left(x_{2}, y_{2}\right)$ with the same sequence of differences of ranks between corresponding elements. We do not need this dummy element of rank 0 since the first difference is actually a rank. It thus suffices to show that we can express the predicate

$$
D((u, v),(z, t))=d
$$

meaning that the difference of the ranks of $(u, v)$ and $(z, t)$ is equal to $d \leq$ last and the predicate

$$
R(u, v)=\rho
$$

meaning that the rank of $(u, v)$ is equal to $\rho \leq$ last. Since the first component of each element has value bounded by $k$, the set $\{(x, y) \mid(u, v)<(x, y) \leq(z, t)\}$ is the union, for $1 \leq i \leq k$, of

$$
X_{i}=\{(i, y) \mid(u, v)<(i, y) \leq(z, t)\} .
$$

As the definition of counting quantifier requires a nonzero value, we must select among the $X_{i}$ 's those which are nonempty. In order to express (4) we are thus led to consider the 
disjunction over all possible sequences $1 \leq i_{1}<\cdots<i_{r} \leq k$ of the following predicates (but at most one sequence is satisfied):

$$
\begin{gathered}
\exists d_{1} \cdots \exists d_{r}\left(\left(d=\sum_{1 \leq s \leq r} d_{s}\right) \wedge \bigwedge_{s=1}^{r} \exists_{y}^{d_{s}}\left((u, v)<\left(i_{s}, y\right) \leq(z, t)\right)\right) \wedge \\
\bigwedge_{i \neq i_{1}, \ldots, i_{r}} \neg \exists y((u, v)<(i, y) \leq(z, t)) .
\end{gathered}
$$

The predicate (5) can be dealt with similarly:

$$
\begin{gathered}
\exists \rho_{1} \cdots \exists \rho_{r}\left(\left(\rho=\sum_{1 \leq s \leq r} \rho_{s}\right) \wedge \bigwedge_{s=1}^{r} \exists_{y}^{\rho_{s}}\left(\left(i_{s}, y\right) \leq(u, v)\right)\right) \wedge \\
\bigwedge_{i \neq i_{1}, \ldots, i_{r}} \neg \exists y((i, y) \leq(u, v)) .
\end{gathered}
$$

Concerning the property (2), we proceed in the same manner by decomposing all pairs of elements according to the value of their first component, since this value is bounded by $k$. We use a formula in FOC $[+]$ which has $2 p$ variables, namely $x_{2}, y_{2}, \ldots, x_{p}, y_{p}, x, y$ and which is again a disjunction over all possible sequences $1 \leq i_{1}<\cdots<i_{r} \leq k$ of the following formulas:

$$
\begin{aligned}
& \exists d_{1} \ldots \exists d_{r} \exists\left(u_{1}, v_{1}\right) \cdots \exists\left(u_{r}, v_{r}\right)\left[\left(u_{r}, v_{r}\right)=(x, y) \wedge\left(R\left(u_{1}, v_{1}\right)=d_{1}\right) \wedge\right. \\
& \left.\quad\left(\bigwedge_{s=1}^{r-1} D\left(\left(u_{s}, v_{s}\right),\left(u_{s+1}, v_{s+1}\right)\right)=d_{s+1}\right) \wedge \bigwedge_{s=1}^{r} \exists_{y}^{d_{s}} \phi\left(\left(i_{s}, y\right),\left(x_{2}, y_{2}\right), \ldots,\left(x_{p}, y_{p}\right)\right)\right] \wedge \\
& \bigwedge_{i \neq i_{1}, \ldots, i_{r}} \neg \exists y \phi\left((i, y),\left(x_{2}, y_{2}\right), \ldots,\left(x_{p}, y_{p}\right)\right) .
\end{aligned}
$$

We apply this result to the following property of $\mathscr{L}(\mathrm{FOC}[+])$ :

Proposition 5 The class $\mathscr{L}(\mathrm{FOC}[+])$ is closed under all inverse homomorphisms.

Proof We use the preliminary observation of this subsection concerning the classification of morphisms. The first two cases can be treated as in Proposition 3 (counting quantifiers are straightforwardly managed). The erasing morphisms are actually considered in Proposition 8 , since they can be regarded as a particular disjoint shuffle. Therefore it is enough to prove the result for growing homomorphisms. More precisely, we prove that if a language $L$ is expressible in FOC $[+]$ then $g^{-1}(L)$ is also expressible in $\mathrm{FOC}[+]$, where $g$ is a growing morphism defined as $g(a)=a c$ and $g(b)=b$, for every $b \neq a$.

The universe of the structure $h(w)$ can be encoded into the universe $\{1, \ldots,|w|\}$ of $w$ via the $\mathrm{FOC}[+]$ predicate:

$$
\mathrm{U}(x, y) \equiv\left(y=1 \vee\left(Q_{a}(x) \wedge y=2\right)\right)
$$

The rank $\rho(i, j)$ of a pair $(i, j)$ is defined according to the alphabetic ordering and satisfies the condition

$$
\rho(i, j)=i+\left|\left\{x \mid Q_{a}(x) \wedge x<i\right\}\right|+j-1 .
$$


Given a formula $\phi\left(x_{1}, \ldots, x_{n}\right)$ in $\mathrm{FOC}[+]$ and a word $h(w)$, we show how to associate a formula $\phi^{\prime}\left(\left(x_{1}, y_{1}\right), \ldots,\left(x_{n}, y_{n}\right)\right)$ in FOC $[+]$ such that the following holds:

$$
\begin{aligned}
\left(w, \rho^{-1}\left(i_{1}\right), \ldots, \rho^{-1}\left(i_{n}\right)\right) & \models \phi^{\prime}\left(\left(x_{1}, y_{1}\right), \ldots,\left(x_{n}, y_{n}\right)\right) \\
& \Leftrightarrow \\
\left(h(w), i_{1}, \ldots, i_{n}\right) & \models \phi\left(x_{1}, \ldots, x_{n}\right) .
\end{aligned}
$$

We proceed by structural induction starting with the basic predicates.

$$
\text { If } \phi(x) \equiv Q_{b}(x) \text { then }
$$

$$
\phi^{\prime}(x, y) \equiv \begin{cases}Q_{a}(x) \wedge y=1 & \text { if } b=a \\ Q_{a}(x) \wedge y=2 \text { if } b=c \\ Q_{b}(x) \wedge y=1 \text { otherwise, }\end{cases}
$$

and the predicate $x=y+z$ is replaced by the predicate of Proposition 4(i).

With the formula $\neg \phi\left(x_{1}, \ldots, x_{n}\right)$, we associate the formula

$$
\neg \phi^{\prime}\left(\left(x_{1}, y_{1}\right), \ldots,\left(x_{n}, y_{n}\right)\right) \wedge \bigwedge_{1 \leq i \leq n} \mathrm{U}\left(x_{i}, y_{i}\right) .
$$

Concerning the conjunction, assume without loss of generality that the two formulas are $\phi_{1}\left(x_{1}, \ldots, x_{n}, \ldots, x_{n+m}\right)$ and $\phi_{2}\left(x_{n+1}, \ldots, x_{n+m}, \ldots x_{n+m+p}\right)$, i.e., that the common variables occur among the last and first ones in the formulas, respectively. Then, with the formula

$$
\phi_{1}\left(x_{1}, \ldots, x_{n+m}\right) \wedge \phi_{2}\left(x_{n+1}, \ldots, x_{n+m+p}\right)
$$

we associate the formula

$$
\phi_{1}^{\prime}\left(\left(x_{1}, y_{1}\right), \ldots,\left(x_{n+m}, y_{n+m}\right)\right) \wedge \phi_{2}^{\prime}\left(\left(x_{n+1}, y_{n+1}\right), \ldots,\left(x_{n+m+p}, y_{n+m+p}\right)\right) .
$$

Finally, the rule for the counting quantifiers is given in Proposition 4(ii).

\subsection{Concatenation, Shuffle and Kleene Star}

Let us start by considering the closure under concatenation:

Proposition 6 The classes $\mathscr{L}(\mathrm{FO}[<]), \mathscr{L}(\mathrm{FO}[+])$ and $\mathscr{L}(\mathrm{FOC}[+])$ are closed under concatenation, while the class $\mathscr{L}(\mathrm{FO}[+1])$ is not.

Proof As a preliminary observation, we assume that in the formulas $\phi_{1}$ and $\phi_{2}$ defining two languages $L_{1}$ and $L_{2}$, respectively, all quantified variables are expressed in the form $\exists x \leq$ last and $\forall x \leq$ last and that no variable, whether free or bound, is shared by both formulas. We apply the method of relativization: if $u \in L_{1}$ and $v \in L_{2}$ then $u v$ is defined by saying that $u$ satisfies $\phi_{1}$, and that the "translate" of the factor $v$ obtained by shifting it $|u|$ positions to the left satisfies $\phi_{2}$. The new formula is therefore of the form

$$
\psi=\exists \ell\left(\phi_{1}^{\prime} \wedge \phi_{2}^{\prime}\right) .
$$

The case $\mathscr{L}(\mathrm{FO}[<])$ can be treated directly, but the result is a simple consequence of the fact that it is a pseudovariety of languages.

Concerning $\mathscr{L}(\mathrm{FO}[+])$, we apply two different sets of transformations on the formulas with arbitrary free variables according to whether we define the prefix or the suffix of the word $w=u v$ : 
- $\phi_{1}^{\prime}$ is obtained from $\phi_{1}$ by replacing last by $\ell$, every occurrence of $Q_{a}(x)$ by $Q_{a}(x) \wedge x \leq$ $\ell$, every occurrence of $z=x+y$ by $z=x+y \wedge(x \leq \ell) \wedge(y \leq \ell) \wedge(z \leq \ell)$. Straightforward substitutions can manage the inductive construction of formulas.

- $\phi_{2}^{\prime}$ is obtained from $\phi_{2}$ by replacing every occurrence of $Q_{a}(x)$ by $Q_{a}(x) \wedge x>\ell$, every occurrence of $z=x+y$ by $z=x+y-\ell \wedge(x>\ell) \wedge(y>\ell) \wedge(z>\ell)$. Straightforward substitutions can manage the inductive construction of formulas.

It suffices to check by structural induction that the following holds

$$
\begin{gathered}
\left(u, i_{1}, \ldots, i_{r}\right) \models \phi_{1} \text { and }\left(v, j_{1}, \ldots, j_{s}\right) \models \phi_{2} \\
\left.\Leftrightarrow u, i_{1}, \ldots, i_{r}, j_{1}+|u|, \ldots, j_{s}+|u|\right) \models \phi_{1}^{\prime} \wedge \phi_{2}^{\prime} .
\end{gathered}
$$

We now deal with the third language class, and assume $\phi_{1}$ and $\phi_{2}$ are FOC $[+]$ formulas:

- $\phi_{1}^{\prime}$ is obtained from $\phi_{1}$ as above with the additional rule

- $\exists_{x_{1}}^{y} \theta\left(x_{1}, \ldots, x_{n}\right)$ replaced by $\exists_{x_{1}}^{y}\left(\theta\left(x_{1}, \ldots, x_{n}\right) \wedge\left(x_{1} \leq \ell\right)\right)$.

- $\phi_{2}^{\prime}$ is obtained from $\phi_{2}$ as above with the additional rule

- $\exists_{x_{1}}^{y} \theta\left(x_{1}, \ldots, x_{n}\right)$ replaced by $\left.\exists t\left(\exists_{x_{1}}^{t} \theta\left(x_{1}, \ldots, x_{n}\right) \wedge\left(x_{1}>\ell\right)\right) \wedge(y=t+\ell)\right)$.

where $t$ is a new variable. With these two modifications, it suffices to verify a formula as (7).

For the last statement, it suffices to observe that the languages $c^{*} a c^{*}$ and $c^{*} b c^{*}$ are in $\mathscr{L}($ FOC $[+1])$ but not their concatenation (see, e.g., [18, Cor. IV.3.4]).

We now turn to the shuffle operation which assigns a finite subset of words to a pair of words by the following recursive definition:

$$
\begin{aligned}
& w \text { Ш } \varepsilon=\varepsilon Ш w=w, \\
& a u Ш b v=b(a u Ш v) \cup a(u Ш b v), \text { with } a, b \in \Sigma \text { and } u, v \in \Sigma^{*} .
\end{aligned}
$$

E.g., if $u=a b a$ and $v=a a$ then $u W v=\left\{a^{3} b a, a^{2} b a^{2}, a b a^{3}\right\}$. This notation extends to languages $A, B \subseteq \Sigma^{*}$ by defining $A \amalg B=\bigcup_{x \in A, y \in B} x \amalg y$. A disjoint shuffle is a shuffle performed between two languages defined over disjoint alphabets.

Proposition 7 The classes $\mathscr{L}(\mathrm{FO}[+1]), \mathscr{L}(\mathrm{FO}[<])$ and $\mathscr{L}(\mathrm{FO}[+])$ are not closed under shuffle.

Proof This assertion is proved via counterexamples. The languages $X=c^{*}$ and $Y=\{a b\}$ are in $\mathscr{L}(\mathrm{FO}[+1])$ but $X \amalg Y$ is not. The languages $X=b^{*}, Y=\left(a^{2} b\right)^{*}$ and $Z=(a b)^{*}$ are in $\mathscr{L}(\mathrm{FO}[<])$ but $(X \amalg Y) \cap Z=\left((a b)^{2}\right)^{*}$ is not. Finally, the languages $X=\left(a^{2}\right)^{*}$ and $Y=b^{*}$ belong to $\mathscr{L}(\mathrm{FO}[+])$ but $X \amalg Y$ does not by [2, Cor. 4.2].

The situation changes for disjoint shuffles:

Proposition 8 The classes $\mathscr{L}(\mathrm{FO}[<])$ and $\mathscr{L}(\mathrm{FOC}[+])$ are closed under disjoint shuffle, while the classes $\mathscr{L}(\mathrm{FO}[+1])$ and $\mathscr{L}(\mathrm{FO}[+])$ are not. 
Proof Let the formulas $\phi_{1}$ and $\phi_{2}$ define the languages $L_{1} \subseteq \Sigma_{1}^{*}$ and $L_{2} \subseteq \Sigma_{2}^{*}$, respectively, with $\Sigma_{1} \cap \Sigma_{2}=\emptyset$. Given $u \in \Sigma_{1}^{*}$ and $v \in \Sigma_{2}^{*}$, let $w=w_{1} \cdots w_{r+s} \in u \amalg v$ with $|u|=r$ and $|v|=s$. For $i=1, \ldots r$, let $f(i)$ be the position in $w$ of the $i$ th letter of $u$, and similarly let $g(j)$ be the position in $w$ of the $j$ th letter of $v$. Example: with $u=a b a, v=d d c d$ and $w=d d a b c d a$ we have $f(1)=3, f(2)=4, f(3)=7$ and $g(1)=1, g(2)=2, g(3)=5, g(4)=6$.

The statement for $\mathrm{FO}[<]$ comes straightforwardly since the syntactic monoid of $L_{1} \amalg L_{2}$ is the direct product of the syntactic monoids of $L_{1}$ and $L_{2}$. Since each of these two syntactic monoids has trivial groups only, so does the direct product.

Concerning FOC $[+]$, we assume without loss of generality that the formulas $\phi_{1}$ and $\phi_{2}$ do not contain standard existential quantifiers since they can be simulated by counting quantifiers, that the symbol last does not occur and that the two sets of their variables, whether free or bound, are disjoint. In order to simplify the notations, we let $Q_{\Sigma_{i}}(x)$ stand for $\bigvee_{a \in \Sigma_{i}} Q_{a}(x)$. We denote by $\phi^{\prime}$ the formula resulting from $\phi$ and we drop the index since for obvious reasons of symmetry, the two formulas are modified according to the same rules. We proceed according to the usual structural induction, by applying the following rules:

- If $\phi(x) \equiv Q_{a}(x)$ then $\phi^{\prime}(x) \equiv Q_{a}(x)$.

- If $\phi(x, y, z) \equiv x=y+z$ then

$$
\begin{aligned}
& \phi^{\prime}(x, y, z) \equiv \exists x^{\prime} \exists y^{\prime} \exists z^{\prime}\left(\left(x^{\prime}=y^{\prime}+z^{\prime}\right) \wedge \exists_{u}^{y^{\prime}}\left(Q_{\Sigma}(u) \wedge u \leq y\right) \wedge\right. \\
& \left.\quad \exists_{u}^{z^{\prime}}\left(Q_{\Sigma}(u) \wedge u \leq z\right) \wedge \exists_{u}^{x^{\prime}}\left(Q_{\Sigma}(u) \wedge u \leq x\right)\right) .
\end{aligned}
$$

- If $\phi \equiv \neg \psi\left(x_{1}, \ldots, x_{n}\right)$ then

$$
\phi^{\prime} \equiv \neg \psi^{\prime}\left(x_{1}, \ldots, x_{n}\right) \wedge \bigwedge_{i=1}^{n} Q_{\Sigma}\left(x_{i}\right) .
$$

- If $\phi \equiv \psi \wedge \theta$ then $\phi^{\prime} \equiv \psi^{\prime} \wedge \theta^{\prime}$

- If $\phi(x) \equiv \exists_{u}^{x} \psi(u)$ then $\phi^{\prime}(x) \equiv \exists_{u}^{x}\left(\psi^{\prime}(u) \wedge Q_{\Sigma}(u)\right)$.

It suffices to verify by structural induction that if $\phi_{1}^{\prime}$ and $\phi_{2}^{\prime}$ are the formulas obtained from $\phi_{1}$ and $\phi_{2}$ by applying the above rules, then we have

$$
\begin{gathered}
\left(u, i_{1}, \ldots, i_{r}\right) \models \phi_{1}\left(x_{1}, \ldots, x_{r}\right) \text { and }\left(v, j_{1}, \ldots, j_{s}\right) \models \phi_{2}\left(y_{1}, \ldots, y_{s}\right) \\
\Leftrightarrow \\
\left(w, f\left(i_{1}\right), \ldots, f\left(i_{r}\right), g\left(j_{1}\right), \ldots, g\left(j_{s}\right)\right) \models \phi_{1}^{\prime}\left(x_{1}, \ldots, x_{r}\right) \wedge \phi_{2}^{\prime}\left(y_{1}, \ldots, y_{s}\right) .
\end{gathered}
$$

To see that $\mathscr{L}(\mathrm{FO}[+1])$ and $\mathscr{L}(\mathrm{FO}[+])$ are not closed under disjoint shuffle, it is enough to check counterexamples provided in Proposition 7 for general shuffle.

Finally, let us consider the closure under Kleene star:

Proposition 9 The classes $\mathscr{L}(\mathrm{FO}[+1]), \mathscr{L}(\mathrm{FO}[<])$ and $\mathscr{L}(\mathrm{FO}[+])$ are not closed under Kleene star.

Proof The counterexample for $\mathscr{L}(\mathrm{FO}[+1])$ and $\mathscr{L}(\mathrm{FO}[<])$ is the subset $\left(a^{2}\right)^{*}$. Now, if $\mathscr{L}(\mathrm{FO}[+])$ were closed under Kleene star, by Proposition 6 the whole class of regular languages would be contained in $\mathscr{L}(\mathrm{FO}[+])$ due to Kleene's Theorem. However, the regular language $\left\{\left.w \in\{a, b\}^{+}|| w\right|_{a} \bmod 2=0\right\}$ does not belong to $\mathscr{L}(\mathrm{FO}[+])$ by [2, Cor. 4.2], a contradiction. 
To the best of our knowledge, the status of $\mathscr{L}(\mathrm{FOC}[+])$ relative to the Kleene star is still open. All we can say is that:

If $\mathscr{L}(\mathrm{FOC}[+])$ were closed under Kleene star, then $\mathrm{TC}^{0}=\mathrm{NC}^{1}$.

Indeed, in that case $\mathscr{L}(\mathrm{FOC}[+]) \subset \mathrm{TC}^{0}$ would be closed under the regular operations and thus it would contain all regular languages. However, as proved in [3], there exists a regular language which is complete for $\mathrm{NC}^{1}$.

\subsection{Quotient}

The left quotient of a language $L$ by a letter $a$, denoted $a^{-1} L$, is the set of words $u$ such that $a u \in L$. In this subsection, "quotient" means left quotient. For obvious reasons of symmetry, the same result holds for right quotients.

Proposition 10 The classes $\mathscr{L}(\mathrm{FO}[+1]), \mathscr{L}(\mathrm{FO}[<]), \mathscr{L}(\mathrm{FO}[+]), \mathscr{L}(\mathrm{FOC}[+])$ are closed under quotient.

Proof We encode the universe of $a w$ into the universe of $w$ as the set of pairs $(x, y)$ which satisfy the predicate

$$
\mathrm{U}(x, y) \equiv((x=1 \wedge y=1) \vee x=2) .
$$

The rank of an element $(i, j)$ of this set relative to the alphabetic ordering is $\rho(i, j)=i+j-1$, and can be defined in the logics $\mathrm{FO}[+]$ and $\mathrm{FOC}[+]$. We encode the predicates $Q_{b}$ and +1 , of the universe of $a w$ into the universe of $w$ as follows, respectively:

$$
\begin{gathered}
P_{b}(x, y) \equiv \begin{cases}(x=1 \wedge y=1) \vee\left(x=2 \wedge Q_{a}(y)\right) & \text { if } b=a \\
x=2 \wedge Q_{b}(y) & \text { otherwise. }\end{cases} \\
\operatorname{Succ}\left(\left(x_{1}, y_{1}\right),\left(x_{2}, y_{2}\right)\right) \equiv\left(x_{1}=y_{1}=y_{2}=1 \wedge x_{2}=2\right) \vee \\
\left(\left(x_{1}=x_{2}=2\right) \wedge\left(y_{2}=y_{1}+1\right)\right) .
\end{gathered}
$$

If $\phi \in \mathrm{FO}[+1]$ defines the language $L$ then the language $a^{-1} L$ is defined by the formula $\phi^{\prime}$ which is obtained by recursively applying the following rules:

- If $\phi(x) \equiv Q_{b}(x)$ then $\phi^{\prime}(x, y) \equiv P_{b}(x, y)$.

- If $\phi\left(x_{1}, x_{2}\right) \equiv x_{2}=x_{1}+1$ then $\phi^{\prime}\left(\left(x_{1}, y_{1}\right),\left(x_{2}, y_{2}\right)\right) \equiv \operatorname{Succ}\left(\left(x_{1}, y_{1}\right),\left(x_{2}, y_{2}\right)\right)$.

- If $\psi\left(x_{1}, \ldots, x_{n}\right) \equiv \neg \phi\left(x_{1}, \ldots, x_{n}\right)$ then

$$
\psi^{\prime}\left(\left(x_{1}, y_{1}\right), \ldots,\left(x_{n}, y_{n}\right)\right) \equiv \neg \phi^{\prime}\left(\left(x_{1}, y_{1}\right), \ldots,\left(x_{n}, y_{n}\right)\right) \wedge \bigwedge_{i=1}^{n} \mathrm{U}\left(x_{i}, y_{i}\right) .
$$

- If $\psi \equiv \phi_{1} \wedge \phi_{2}$ then $\psi^{\prime} \equiv \phi_{1}^{\prime} \wedge \phi_{2}^{\prime}$

- If $\psi\left(x_{1}, x_{2}, \ldots, x_{n}\right) \equiv \exists x_{1} \phi\left(x_{1}, x_{2}, \ldots, x_{n}\right)$ then

$$
\psi^{\prime}\left(\left(x_{1}, y_{1}\right),\left(x_{2}, y_{2}\right), \ldots,\left(x_{n}, y_{n}\right)\right) \equiv \exists x_{1} \exists y_{1} \phi^{\prime}\left(\left(x_{1}, y_{1}\right),\left(x_{2}, y_{2}\right), \ldots,\left(x_{n}, y_{n}\right)\right) \wedge \mathrm{U}\left(x_{1}, y_{1}\right)
$$


The assertion concerning $\mathrm{FO}[<]$ follows from the fact that $\mathscr{L}(\mathrm{FO}[<])$ is a pseudovariety. In order to treat the case $\mathrm{FO}[+]$, we encode the predicate $x+y=z$ by the predicate which asserts that the rank of $\left(z_{1}, z_{2}\right)$ in the alphabetic ordering is the sum of the ranks of $\left(x_{1}, x_{2}\right)$ and $\left(y_{1}, y_{2}\right)$, i.e., $\rho\left(x_{1}, y_{1}\right)+\rho\left(x_{2}, y_{2}\right)=\rho\left(x_{3}, y_{3}\right)$, which is clearly expressible in $\mathrm{FO}[+]$. Then, we have

$$
\begin{aligned}
&\left(w,\left(i_{1}, j_{1}\right), \ldots,\left(i_{n}, j_{n}\right)\right) \models \phi^{\prime}\left(\left(x_{1}, y_{1}\right), \ldots,\left(x_{n}, y_{n}\right)\right) \\
& \Leftrightarrow \\
&\left(a w, \rho\left(i_{1}, j_{1}\right), \ldots, \rho\left(i_{n}, j_{n}\right)\right) \models \phi\left(x_{1}, \ldots, x_{n}\right) .
\end{aligned}
$$

For FOC $[+]$, the only new transformation rule is the one involving $\exists_{x_{1}}^{y} \phi\left(x_{1}, \ldots, x_{n}\right)$. By induction, with the formula $\phi$ for $L$ the formula $\phi^{\prime}\left(\left(x_{1}, y_{1}\right), \ldots,\left(x_{n}, y_{n}\right)\right)$ for $a^{-1} L$ is assigned. Indeed, we replace the formula $\exists_{x_{1}}^{y} \phi\left(x_{1}, \ldots, x_{n}\right)$ with the formula

$$
\psi\left(\left(z_{1}, t_{1}\right),\left(x_{2}, y_{2}\right), \ldots,\left(x_{n}, y_{n}\right)\right)
$$

whose truth value for a fixed assignment $\left(a_{2}, b_{2}\right), \ldots,\left(a_{n}, b_{n}\right)$ can be described as follows: Let $r$ be the number of values $\left(a_{1}, b_{1}\right)$ such that $\phi^{\prime}\left(\left(a_{1}, b_{1}\right),\left(a_{2}, b_{2}\right), \ldots,\left(a_{n}, b_{n}\right)\right)$ holds true. If $r=0, \psi\left(\left(z_{1}, t_{1}\right),\left(a_{2}, b_{2}\right), \ldots,\left(a_{n}, b_{n}\right)\right)$ is a contradiction, otherwise it assumes the value true for the unique pair $\left(a_{1}, b_{1}\right)$ such that $\rho\left(a_{1}, b_{1}\right)=r$. The implementation of $\psi$ is given in Proposition 4(ii).

\subsection{Conjugacy}

We recall that two words $x$ and $y$ are conjugate if there exist $u$ and $v$ such that $x=u v$ and $y=v u$. More generally, the conjugate of a language $L$ is the set of all conjugates of the words it contains, i.e., it is the set $\operatorname{CONJ}(L)=\left\{v u \in \Sigma^{*} \mid u v \in L\right\}$.

Proposition 11 The classes $\mathscr{L}(\mathrm{FO}[+1]), \mathscr{L}(\mathrm{FO}[<]), \mathscr{L}(\mathrm{FO}[+]), \mathscr{L}(\mathrm{FOC}[+])$ are closed under conjugacy.

Proof A language is expressible in $\mathrm{FO}[+1]$ if and only if it is locally threshold testable [18], i.e., for some integers $k$ and $T$ it is a union of classes of an equivalence relation $u \sim v$ defined by the following conditions:

- $u$ and $v$ have the same prefix and the same suffix of length $k-1$,

- for all $w$ of length $k$, either $|u|_{w}=|v|_{w}<T$ or $|u|_{w},|v|_{w} \geq T$ holds.

It thus suffices to consider a single equivalence class consisting of words of length greater than $k$, i.e. to fix $k, T \in \mathbb{N}$ and $p, s \in \Sigma^{k-1}$, a mapping $f: \Sigma^{k} \rightarrow\{0, \ldots, T\}$, and to consider the language of the words $u$ satisfying

$$
u \in p \Sigma^{*} \cap \Sigma^{*} s \text { and, for all } w \in \Sigma^{k},|u|_{w}=f(w) \text { if } f(w)<T \text { or else }|u|_{w} \geq T .
$$

Let us introduce the following definition. A word $w$ is a cyclic occurrence of $u$ if $u=x w y$ holds or if there exists a factorization $w=w_{1} w_{2}$, with $w_{1}, w_{2} \neq \varepsilon$, and a word $x$ such that $u=w_{2} x w_{1}$ holds. The cyclic occurrence starts (resp., ends) at position $k$ if $|x|=k$ in the first case and if $\left|w_{2} x\right|=k$ in the second case (resp., if $|x w|=k$ in the first case and if $\left|w_{2}\right|=k$ in the second case). Moreover, it avoids position $\ell$ if it does not contain the $\ell$ th letter of $u$. Then, the set of conjugates of this equivalence class is defined by a formula which says that for some integer $\ell$, the word has a cyclic occurrence of $p$ starting at position $\ell$, a cyclic occurrence of $s$ ending at position $\ell$ and such that for all words $w$ of length $k$, it has exactly 
$f(w)$ cyclic occurrences $w$ avoiding $\ell$ if $f(w)<T$ or at least $T$ cyclic occurrences avoiding $\ell$ otherwise.

The result concerning $\mathrm{FO}[<]$ is well-known. We briefly recall it for the sake of self containment. Consider a deterministic aperiodic automaton ${ }^{1}$ recognizing the language $L$, with $q_{0}$ as initial state and $F$ as set of final states. Then the set of conjugates of $L$ is the union of the languages $Y_{q} Z_{q}$, where $Y_{q}$ is the set of words recognized by the automaton with $q$ as initial state and $F$ as set of final state and $Z_{q}$ is the set of words recognized by the automaton with $q_{0}$ as initial state and where the set of final states reduces to $q$. These automata are aperiodic, thus they recognize star-free languages. We conclude by recalling that, by definition, star-free languages are closed under union and concatenation.

For the logic $\mathrm{FO}[+]$, we proceed as follows. Given a formula $\phi$ defining a language $L$, we construct a formula $\exists t \phi^{\prime}(t)$ defining the conjugate of $L$. The variable $t$ under the existential quantifier must be interpreted as the shift of a word in $L$. Indeed, consider the following function describing, for all fixed values of the variable $t$, a bijection of $\{1, \ldots$, last $\}$ into itself which defines where the position of a letter in $u v$ maps in $v u$ :

$$
f(x, t)= \begin{cases}x+\text { last }-t & \text { if } x \leq t \\ x-t & \text { otherwise. }\end{cases}
$$

Observe that this correspondence is definable in $\mathrm{FO}[+]$.

From a formula $\phi\left(x_{1}, \ldots, x_{n}\right)$ in $\mathrm{FO}[+]$, we define a formula $\phi^{\prime}\left(x_{1}, \ldots, x_{n}, t\right)$ with the property

$$
\begin{aligned}
& \left(u v, i_{1}, \ldots, i_{n}\right) \models \phi\left(x_{1}, \ldots, x_{n}\right) \\
& \Leftrightarrow \\
& \left(v u, f\left(i_{1},|u|\right), \ldots, f\left(i_{n},|u|\right),|u|\right) \models \phi^{\prime}\left(x_{1}, \ldots, x_{n}, t\right)
\end{aligned}
$$

by applying the usual structural induction (we assume the variable $t$ does not occur in the formula $\phi$ ):

- If $\phi(x) \equiv Q_{a}(x)$ then $\phi^{\prime}(x, t) \equiv Q_{a}(f(x, t))$.

- If $\phi(x, y, z) \equiv z=x+y$ then $\phi^{\prime}(x, y, z, t) \equiv f(z, t)=f(x, t)+f(y, t)$.

- If $\phi\left(x_{1}, \ldots, x_{n}\right) \equiv \phi_{1}\left(y_{1}, \ldots, y_{p}\right) \wedge \phi_{2}\left(z_{1}, \ldots, z_{m}\right)$, where $x_{1}, \ldots, x_{n}$ is the set consisting of the variables of $\phi_{1}$ and of $\phi_{2}$, then $\phi^{\prime}\left(x_{1}, \ldots, x_{n}, t\right) \equiv \phi_{1}^{\prime}\left(y_{1}, \ldots, y_{p}, t\right) \wedge \phi_{2}^{\prime}\left(z_{1}, \ldots, z_{m}, t\right)$.

- If $\phi \equiv \neg \psi$ then $\phi^{\prime} \equiv \neg \psi^{\prime}$.

- If $\phi \equiv \exists x_{1} \psi\left(x_{1}, x_{2}, \ldots, x_{n}\right)$ then $\phi^{\prime} \equiv \exists x_{1} \psi^{\prime}\left(x_{1}, x_{2}, \ldots, x_{n}\right)$.

So, if $L$ is defined by the closed formula $\phi$ then its conjugate is defined by the formula $\exists t \phi^{\prime}(t)$.

We now turn to the logic FOC $[+]$. It suffices to show how to deal with the counting quantifier $\exists_{x_{1}}^{y} \phi\left(x_{1}, \ldots, x_{n}\right)$. This expression is recursively transformed into

$$
\begin{aligned}
& \exists y_{1} \exists y_{2}\left(f(y, t)=f\left(y_{1}, t\right)+f\left(y_{2}, t\right)\right) \wedge \\
& \quad \exists_{x_{1}}^{y_{1}}\left(\phi^{\prime}\left(x_{1}, \ldots, x_{n}, t\right) \wedge\left(x_{1} \leq \text { last }-t\right)\right) \wedge \\
& \quad \exists_{x_{1}}^{y_{2}}\left(\phi^{\prime}\left(x_{1}, \ldots, x_{n}, t\right) \wedge\left(x_{1}>\text { last }-t\right)\right) .
\end{aligned}
$$

Similarly to $\mathrm{FO}[+]$, if a language $L$ is defined by a closed formula $\phi$ then its conjugate is defined by the formula $\exists t \phi^{\prime}(t)$.

\footnotetext{
1 A deterministic automaton is aperiodic if and only if there exists $n>0$ such that $q \cdot w^{n}=q \cdot w^{n+1}$, for any state $q$ and any word $w$.
} 
4.5 Commutative Image

The commutative image of a language $L$ is the language

$$
\operatorname{COMM}(L)=\left\{x_{1} \cdots x_{n} \in \Sigma^{*} \mid x_{i_{1}} \cdots x_{i_{n}} \in L \text { and }\left\{i_{1}, \ldots, i_{n}\right\}=\{1, \ldots, n\}\right\} .
$$

Proposition 12 The classes $\mathscr{L}(\mathrm{FO}[+1]), \mathscr{L}(\mathrm{FO}[<])$ and $\mathscr{L}(\mathrm{FO}[+])$ are not closed under commutative image.

Proof For $\mathscr{L}(\mathrm{FO}[+1])$ and $\mathscr{L}(\mathrm{FO}[<])$, it suffices to see that $\mathrm{COMM}\left((a b)^{*}\right)$ is not a regular language. Now, let $L=\left\{a b a b^{2} a b^{3} a \cdots a b^{i} a \cdots a b^{k} a \mid k>0\right\}$. By the proof of Proposition 2, we have that $L \in \mathrm{FO}[+]$. Consider the language $L^{\prime}=\operatorname{COMM}(L) \cap a^{*} b^{*}=\left\{a^{n} b^{\frac{(n-1) n}{2}} \mid n>1\right\}$. If $\mathscr{L}(\mathrm{FO}[+])$ were closed under commutative image, then $L^{\prime}$ should be semilinear by Theorem 2 , a contradiction.

\subsection{Reversal}

Given a word $x=x_{1} \cdots x_{n}$, with $x_{i} \in \Sigma$, its reversal is the word $x^{R}=x_{n} \cdots x_{1}$ (with $\varepsilon^{R}=\varepsilon$ ). The reversal of a language $L$ is the language $L^{R}=\left\{x^{R} \mid x \in L\right\}$.

Proposition 13 The classes $\mathscr{L}(\mathrm{FO}[+1]), \mathscr{L}(\mathrm{FO}[<]), \mathscr{L}(\mathrm{FO}[+]), \mathscr{L}(\mathrm{FOC}[+])$ are closed under reversal.

Proof Let $\phi$ be a formula defining a language in $\mathscr{L}(\mathrm{FO}[+1]), \mathscr{L}(\mathrm{FO}[<]), \mathscr{L}(\mathrm{FO}[+])$ and $\mathscr{L}(\mathrm{FOC}[+])$, respectively. An $\mathrm{FO}[+1]$ formula for $L^{R}$ is obtained from $\phi$ by replacing each occurrence of $x=y+1$ by $y=x+1$. Concerning $\mathscr{L}(\mathrm{FO}[<])$, it suffices to replace $x<y$ by $y<x$. Concerning $\mathscr{L}(\mathrm{FO}[+])$, we substitute $x+y-$ last $-1=z$ for each occurrence of $x+y=z$ and, in addition for FOC $[+]$, we substitute $\exists_{x_{1}}^{z} \phi\left(x_{1}, \ldots, x_{p}\right) \wedge(z+y=$ last +1$)$ for $\exists_{x_{1}}^{y} \phi\left(x_{1}, \ldots, x_{p}\right)$.

\section{An Application: Dyck Languages}

From [1], we know that the Dyck languages are in $\mathscr{L}(\mathrm{FOC}[+])$, while from [14] we get that they do not belong to $\mathscr{L}(\mathrm{FO}[+])$. As an application of closure properties in the previous section, we are now going to provide an alternative proof of this latter fact.

The following preliminary notions are useful. Let $A$ be a finite set of opening parentheses and let $\bar{A}$ be the set of (one-to-one) corresponding closing parentheses. Set $T=A \cup \bar{A}$; a word in $T^{*}$ is correctly (or well) parenthesized if: (i) any opening parenthesis $a$ is followed by a corresponding closing parenthesis $\bar{a}$, and (ii) if parenthesis $a^{\prime}$ follows $a$, then $a^{\prime}$ is closed before $a$. The Dyck language $D_{T}$ is the set of correctly parenthesized words in $T^{*}$.

The majority function $\mathscr{M}_{\sigma, \bar{\sigma}}:\{\sigma, \bar{\sigma}\}^{*} \rightarrow\{0,1\}$ and the equality function $\mathscr{E}_{\sigma, \bar{\sigma}}:\{\sigma, \bar{\sigma}\}^{*} \rightarrow$ $\{0,1\}$ are defined, respectively, as:

$$
\mathscr{M}_{\sigma, \bar{\sigma}}(x)=\left\{\begin{array}{ll}
1 & \text { if }|x|_{\sigma}>|x|_{\bar{\sigma}} \\
0 & \text { otherwise }
\end{array} \quad \mathscr{E}_{\sigma, \bar{\sigma}}(x)= \begin{cases}1 & \text { if }|x|_{\sigma}=|x|_{\bar{\sigma}} \\
0 & \text { otherwise }\end{cases}\right.
$$

We also need the notion of $\mathrm{AC}^{0}$-reduction between problems $[18,19]$. Informally, a problem $P$ is $\mathrm{AC}^{0}$-reducible to a problem $P^{\prime}$ whenever $P$ can be solved by a family of polynomial size, constant depth, unbounded fan-in AND/OR/NOT-circuits with oracle gates for $P^{\prime}$. In this case, it is easy to see that $P^{\prime} \in \mathrm{AC}^{0}$ implies $P \in \mathrm{AC}^{0}$ as well. 
Theorem 5 The Dyck language $D_{\{a, \bar{a}\}}$ does not belong to $\mathscr{L}(\mathrm{FO}[+])$.

Proof We first prove that $\mathscr{E}_{a, \bar{a}}$ is not in $\mathrm{AC}^{0}$. Indeed, since $\mathscr{M}_{a, \bar{a}}$ is not in $\mathrm{AC}^{0}$ (see, e.g., [7]), it suffices to show that $\mathscr{M}_{a, \bar{a}}$ is $\mathrm{AC}^{0}$-reducible to $\mathscr{E}_{a, \bar{a}}$. Consider $x=x_{1} \cdots x_{n} \in\{a, \bar{a}\}^{n}$. To compute $\mathscr{M}_{a, \bar{a}}(x)$, we build an $\mathrm{AC}^{0}$-circuit $C_{n}$ containing a first layer of oracle gates $O_{0}, \ldots, O_{\left\lfloor\frac{n}{2}\right\rfloor}$ for $\mathscr{E}_{a, \bar{a}}$. As input to $O_{i}$, we give the word $w(i)=a^{i+(n \bmod 2)} x_{i+1} \cdots x_{n}$. If there is an oracle $O_{i}$ yielding 1 , we have that $|x|_{a} \leq|w(i)|_{a}=|w(i)|_{\bar{a}} \leq|x|_{\bar{a}}$. On the contrary, if all $O_{i}$ 's yield 0 , we get that $|x|_{a}>|x|_{\bar{a}}$. Thus, we can complete $C_{n}$ by plugging all the outputs of $O_{i}$ 's into an OR gate whose output, in turn, is sent to a final NOT gate.

Let us now turn to the Dyck language. First of all, it is not difficult to verify that

$$
\operatorname{CONJ}\left(D_{\{a, \bar{a}\}}\right)=\left\{\left.w \in\{a, \bar{a}\}^{*}|| w\right|_{a}=|w|_{\bar{a}}\right\} .
$$

Now, assume by contradiction that $D_{\{a, \bar{a}\}} \in \mathscr{L}(\mathrm{FO}[+])$. Proposition 11 says that $\mathscr{L}(\mathrm{FO}[+])$ is closed under conjugation, thus implying that $\operatorname{CONJ}\left(D_{\{a, \bar{a}\}}\right) \in \mathscr{L}(\mathrm{FO}[+])$ as well. However, we have $w \in \operatorname{CONJ}\left(D_{\{a, \bar{a}\}}\right)$ if and only if $\mathscr{E}_{a, \bar{a}}(w)=1$. Since $\mathscr{E}_{a, \bar{a}}$ is not in $\mathrm{AC}^{0}$ and since $\mathscr{L}(\mathrm{FO}[+]) \subset \mathrm{AC}^{0}$, we get the result.

Theorem 6 The Dyck language $D_{T}$ does not belong to $\mathscr{L}(\mathrm{FO}[+])$.

Proof Let $T=A \cup \bar{A}$ and $a \in A$ a type of parentheses of $D_{T}$. By contradiction, suppose there exists an $\mathrm{FO}[+]$ formula $\phi$ for $D_{T}$, and construct the formula $\phi^{\prime}=\phi \wedge \forall x\left(Q_{a}(x) \vee Q_{\bar{a}}(x)\right)$. Clearly, $\phi^{\prime}$ is an $\mathrm{FO}[+]$ formula for the subset of $D_{T}$ consisting of the well parenthesized words over the alphabet $\{a, \bar{a}\}$, namely $D_{\{a, \bar{a}\}}$. This contradicts Theorem 5 .

\section{References}

1. D.A. Mix Barrington and J. Corbett. On the relative complexity of some languages in NC. Information Processing Letters, 32:251-256, 1989.

2. D.A. Mix Barrington, N. Immerman, C. Lautemann, N. Schweikardt and D. Thérien. First-order expressibility of languages with neutral letters or: The Crane Beach conjecture. J. Comput. Syst. Sci., 70:101-127, 2005.

3. D.A. Mix Barrington, K.J. Compton, H. Straubing and D Thérien. Regular languages in NC ${ }^{1}$. J. Comput. Syst. Sci., 44(3):478-499, 1992.

4. J.R. Büchi. Weak second order arithmetic and finite automata. Zeitschrift für Mathematische Logik und Grundlagen der Mathematik, 6:66-92, 1960.

5. N. Chomsky and M.P. Schützenberger. The algebraic theory of context-free languages. In P. Braffort and D. Hirschberg, Eds., Computer Programming and Formal Systems, pp. 118-161. North Holland, 1963.

6. K. Etessami. Counting quantifiers, successor relations, and logarithmic space. J. Comput. Syst. Sci., 54(3):400-411, 1997.

7. M. Furst, J.B. Saxe and M. Sipser. Parity, circuits, and the polynomial-time hierarchy. Mathematical Systems Theory, 17:13-27, 1984.

8. S. Ginsburg. The Mathematical Theory of Context-Free Languages. McGraw-Hill, 1966.

9. S. Ginsburg and E.H. Spanier. Bounded ALGOL-like languages. Trans. Amer. Math. Soc., 113:333-368, 1964.

10. M.A. Harrison. Introduction to Formal Languages. Addison-Wesley, 1978.

11. O. Ibarra. Simple matrix grammars. Information and Control, 17:359-394, 1970.

12. O. Ibarra. A note on semilinear sets and bounded-reversal multihead pushdown automata. Information Processing Letters, 3:25-28, 1974.

13. O. Ibarra, T. Jiang and B. Ravikumar. Some subclasses of context-free languages in $\mathrm{NC}^{1}$. Information Processing Letters, 29:111-117, 1988.

14. D. Robinson. Parallel algorithms for group word problems. Doctoral Dissertation, Mathematics Dept., University of California, San Diego, 1993. 
15. V. Roychowdhury, K.-Y. Siu and A. Orlitsky. Neural models and spectral methods. In: V. Roychowdhury, K.-Y. Siu, and A. Orlitsky, Eds., Theoretical advances in Neural Computation and Learning, pp. 3-36. Kluwer Academic, 1994.

16. W.L. Ruzzo. On uniform circuit complexity. J. Comput. Syst. Sci., 22:365-383, 1981.

17. N. Schweikardt. Arithmetic, first-order logic, and counting quantifiers. ACM Trans. Comput. Log., 6(3):634-671, 2005

18. H. Straubing. Finite Automata, Formal Logic, and Circuit Complexity. Birkhäuser, 1994

19. I. Wegener. The Complexity of Boolean Functions. Teubner, 1987. 